\title{
Partial Internal Control Recovery on 1-D Klein-Gordon Systems
}

\author{
Iwan Pranoto \\ Faculty of Mathematics and Natural Sciences, \\ Institut Teknologi Bandung, Jl. Ganesha No. 10 Bandung 40132
}

\begin{abstract}
In this exposition, a technique to recover internal control on a distributed parameter system is reported. The system is described by 1-D KleinGordon partial differential equation with a time-varying parameter. We would like to recover the internal control applied to the system if we know some limited information about the output. We use a method called sentinel method to recover the internal control. It involves some construction of a linear functional, and we show that this construction relates closely to the exact controllability problem.
\end{abstract}

Keywords: controllability; distributed parameter system; Klein-Gordon; recovery.

\section{$1 \quad$ Introduction}

Let $\Omega$ stand for the open unit interval $(0,1) \subseteq \mathbb{R}$. The symbol $\mathcal{N}$ denotes the distributed parameter system governed by a non-autonomous 1-D Klein-Gordon equation

$$
L(y)=y_{t t}-y_{x x}+\alpha(t) y=u \text { in } \Omega \times(0, T)
$$

This system covers the string or wave equation and the well-known KleinGordon equation, because if we set $\alpha \equiv 0$, we will obtain the standard wave equation. If we set $\alpha \equiv 1$, we obtain the standard Klein-Gordon equation.

We assume the original system $\mathcal{N}$ has a homogenous boundary condition:

$$
y(0, t)=y(1, t)=0
$$

The symbol $u$ denotes the internal control. We call it internal control, since it affects the system through some internal part of the domain $\Omega$. This internal part, where the support of $u$ lies, is denoted by $\omega$. The subset $\omega$ is a relatively open subset of $\Omega$. Thus, $\operatorname{supp}(u) \subset \omega \times(0, T)$.

We assume the control space is the Hilbert space $\boldsymbol{u}=L^{2}(\omega \times(0, T))$. Thus, $u$ lives in $\boldsymbol{u}$. To make the notation simpler, we extend every internal control in 
$\boldsymbol{u}=L^{2}(\omega \times(0, T))$ to all of $\Omega \times(0, T)$, by assigning $u(x, t)=0$ for $(x, t) \notin \omega \times(0, T)$, and we denote the extended function as $u$ as well. Moreover, we assume that the control $u$ lies in an open $\delta$-ball $B(c, \delta)$ centered at some known $c \in \boldsymbol{U}$, for some $\delta>0$. Thus,

$$
u \in B(c, \delta)=\{\varphi \in \mathcal{U}|| \varphi-c \mid<\delta\}
$$

where $|\cdot|$ denotes the $L^{2}$-norm.

The initial conditions are

$$
y(x, 0)=y_{0}(x) \text { and } y_{t}(x, 0)=y_{1}(x)
$$

We assume that $\left(y_{0}, y_{1}\right)$ belongs to an open $\delta$-ball $B\left(\left(b_{0}, b_{1}\right), \delta\right)$ in the Hilbert space $V=H_{0}^{1}(\Omega) \times L^{2}(0,1)$, for some $\left(b_{0}, b_{1}\right) \in V$.

We are able to observe the dynamics of the system $\mathcal{N}$ through the output function

$$
z(t)=\left.\partial_{x} y(x, t)\right|_{x=1} \text { where } t \in(0, T) .
$$

Thus, one may observe the system only through the derivative of the displacement $y$ on only one extreme of the interval, that is at $x=1$. It is shown that this limited information about the system suffices to partially recover the control $u$, provided some conditions are satisfied.

Because we work with linear systems, the system depends linearly on the initial condition and the control. This implies that the value of $\delta$ does not matter on the existence of the solution, as long as it is positive. This makes sure the variables $\lambda, \tau_{0}, \tau_{1}$ lie in $(-1,1)$.

The problem to recover control from some information on the output was invented by Lions [1,2]. He studied conservative equations like wave and plate equations. If some conditions are satisfied, one can recover the control, or at least recover some information about the control $u$. This process, by observing $z$ and obtaining some information of the internal control $u$, is called partial internal control recovery. In our previous paper Pranoto [3], we extend the above results to include time-variant Klein-Gordon system, where the dimension is higher than 2. It is also shown that the above problem is closely related to the exact controllability problem. In this paper, we complete the results to include the 1-D case. Therefore, now it is true that for any dimension, 
one may recover the internal control of the Klein-Gordon system, provided some conditions are satisfied. The brief summary of the results have been outlined in Pranoto [4].

It is a well known fact that the exact controllability problem of Distributed Parameter System is closely related to the uniqueness property. One such property, for example, is the fact that if the normal derivative of the solution of wave equation with homogenous Dirichlet condition vanishes on the boundary, then the solution is trivial. The exact controllability in this work needs a stronger uniqueness property. We need some inequality, that we call observability inequality. The name observability may become clear later.

The exact controllability of distributed parameter systems usually is studied for linear systems. However, some researchers succeed to generalize the approaches and apply them to semilinear cases, such as in Zuazua [5,6]. Methods for approximating the exact control are proposed. For example, one should consult Zuazua [7,8].

The problem to recover internal control discussed here has many applications. For example, if one interprets the internal control as the source of pollution in ecological environment, this method means that we can detect the location of the pollution source and its intensity by observing merely on some part of the boundary. This is one motivation for studying this problem. See for example Acheli et al [9]. While this paper focuses on the partial recovering of the internal control in an infinite-dimensional system, Fliess et al [10] estimate the state variable of non-linear systems.

\section{Boundary Sentinel}

We assume that $u$ lies in the open $\delta$-ball centered at some $c$. Therefore, one can represent

$$
u=c+\lambda \bar{c}
$$

for some $\lambda \in(-1,1)$ and $c \in B(0, \delta) \subset L^{2}(0, T)$. Similarly, one writes the initial data as

$$
y_{0}=b_{0}+\tau_{0} \bar{b}_{0} \text { and } y_{1}=b_{1}+\tau_{1} \bar{b}_{1}
$$

where $\tau_{0}, \tau_{1} \in(-1,1)$ and $\left(\bar{b}_{1}, \bar{b}_{0}\right) \in B((0,0), \delta) \subset V$.

We define a new system $\boldsymbol{M}$ : 


$$
\begin{aligned}
& L(w)=0 \text { in } \Omega \times(0, T) \\
& w(0, t)=0 \text { and } w(1, t)=\sigma(t)
\end{aligned}
$$

where $\sigma \in L^{2}(0, T)$. The above boundary value problem has the following terminal conditions

$$
w(x, T)=0 \text { and } w_{t}(x, T)=0 \text { for } x \in \Omega
$$

Therefore, the system $\boldsymbol{M}$ is a problem with homogeneous final conditions, but it has a non-homogeneous boundary condition.

We next consider a functional

$$
\mathcal{S}\left(\lambda, \tau_{0}, \tau_{1}\right)=\int_{0}^{T} \sigma(t) z\left(t ; \lambda, \tau_{0}, \tau_{1}\right) d t
$$

where $z\left(t ; \lambda, \tau_{0}, \tau_{1}\right)$ is the output of $\mathcal{N}$, with initial conditions

$$
y_{0}=b_{0}+\tau_{0} \bar{b}_{0} \text { and } y_{1}=b_{1}+\tau_{1} \bar{b}_{1} .
$$

and control

$$
u=c+\lambda \bar{c} .
$$

Thus, $y\left(x, t ; \lambda, \tau_{0}, \tau_{1}\right)$ evaluated at $x=1$ Since we want to recover $u$, we have to make the functional we are going to observe depend only on the internal control $u$. Thus, the functional $S$ must be insensitive to the variation of the initial data. We therefore must impose the following conditions

$$
\partial \tau_{0} S(0,0,0)=\partial \tau_{1} S(0,0,0)=0 .
$$

Thus, by the definition of $z$ in (2), we have the following two identities

$$
\begin{aligned}
& \int_{0}^{T} \sigma(t) \partial_{\tau_{0}} \partial_{x} y(1, t ; 0,0,0) d t=0 \\
& \int_{0}^{T} \sigma(t) \partial_{\tau_{1}} \partial_{x} y(1, t ; 0,0,0) d t=0
\end{aligned}
$$

One may check that $\partial_{\tau_{0}} y$ satisfies the following problem: 


$$
\begin{aligned}
& L\left(\partial_{\tau_{0}} y\right)=0 \text { in } \Omega \\
& \partial_{\tau_{0}} y=0 \text { on } x=0 \text { and } x=1, \\
& \partial_{\tau_{0}} y(x, 0)=\bar{b}_{0} \text { and } \partial_{\tau_{0}} y_{t}(x, 0)=0 .
\end{aligned}
$$

So, if we multiply (3) by $\partial_{\tau 0} y$ and then integrate it over $\Omega \times(0, T)$, we find

$$
\begin{aligned}
0 & =\int_{\Omega \times(0, T)}\left(L(w) \partial_{\tau_{0}} y-L\left(\partial_{\tau_{0}} y\right) w\right) d x d t \\
& =\int_{0}^{T} w(1, t) \partial_{\tau_{0}} \partial_{x} y(1, t ; 0,0,0) d t-\left\langle w_{t}(x, 0), \bar{b}_{0}\right\rangle+(w(x, 0), 0) \\
& =-\left\langle w_{t}(x, 0), \bar{b}_{0}\right\rangle+(w(x, 0), 0) \\
& =-\left\langle w_{t}(x, 0), \bar{b}_{0}\right\rangle
\end{aligned}
$$

for every $\bar{b}_{0}$. We use the Green's formula and the boundary condition of the system $\boldsymbol{M}$ to obtain the above identity. The first term in (6) vanishes, because of the condition (5). Next, the term $-\left\langle w_{t}(x, 0), \bar{b}_{0}\right\rangle$ in (7) is the pairing between $H_{0}^{1}$ and its dual $H^{-1}$, and the other term is the usual $L^{2}$ inner product.

By the non-degeneracy of the form $\langle\cdot, \cdot\rangle$, the fact that (8) is zero for every $\bar{b}_{0}$ implies

$$
w_{t}(x, 0)=0
$$

Similarly, if we multiply (3) by $\partial_{\tau_{1}} y$ and integrate over $\Omega \times(0, T)$, we obtain

$$
w(x, 0)=0
$$

This means, in order to make $S$ independent on the value of $\tau_{0}$ and $\tau_{1}$, the boundary control $\sigma$ must steer the system $\boldsymbol{M}$ from the equilibrium initial state to the equilibrium state again at time $T$. Such boundary control $\sigma$ satisfying (9) and (10) is called boundary sentinel. It is clear that such $\sigma$ exists, since at least $\sigma \equiv 0$ drives equilibrium state to itself, i.e. without moving at all. However, such $\sigma$ is not useful, because the functional $S$ will be zero all the time. Thus, using the trivial sentinel, we will not be able to observe the dynamic of the system. Hence, we must find a sentinel $\sigma$ which is not trivial. 
The proposition below shows that there exists a non-trivial boundary sentinel. Moreover, from the constructive proof of the proposition, one could imply that there are infinitely many boundary sentinels.

Proposition 1. If a satisfies the following conditions:

A1: There is an $\epsilon \in(0,1)$, such that $|\alpha(t)| \leq(1-\epsilon) \pi^{2}$ for every $t$;

$\mathrm{A} 2: \operatorname{Var}(\alpha)<\infty$, where $\operatorname{Var}(\alpha)$ denotes the variation of the function $\alpha$ on $(0, \infty)$,

then for any $e \in L^{2}\left(0, T^{\prime}\right)$, there exist $T^{*}>0$ and $f \in L^{2}\left(T^{\prime}, T^{*}\right)$, such that the concatenate $e * f$ is a boundary sentinel.

The proof of the above proposition relies primarily on the validity of some inequality, called observer inequality. We are interested if there are positive constants $T, k_{T}, K_{T}$ such that the following uniqueness (and regularity) inequality

$$
k_{T}\left\|\left(v_{0}, v_{1}\right)\right\|_{H_{0}^{1} \times L^{2}}^{2} \leq \int_{0}^{T}\left(\partial_{x} v(1, t)\right)^{2} d t \leq K_{T}\left\|\left(v_{0}, v_{1}\right)\right\|_{H_{0}^{1} \times L^{2}}^{2}
$$

is true for all $\left(v_{0}, v_{1}\right) \in H_{0}^{1} \times L^{2}$. Here, $v$ is the solution of the dual system:

$$
\begin{aligned}
& L(v)=0 \text { in } \Omega \times(0, T) \\
& v(0, t)=v(1, t)=0 \text { for all } t \\
& v(x, 0)=v_{0}(x), \quad v_{t}(x, 0)=v_{1}(x) .
\end{aligned}
$$

The above inequality is analogous to the Kalman's rank observability condition utilized widely in the finite dimensional linear system theory. More precisely, let us consider a linear system

$$
\dot{x}=A x+B u \quad x \in \mathbb{R}^{n}
$$

with initial condition $x(0)=x_{0}$. The above system has a dual system

$$
\dot{v}=-A^{T} v \quad v \in \mathbb{R}^{n}
$$

together with an output map

$$
z=B^{T} v
$$


If one multiplies $x$ with $v$ and partially integrate it over $[0, T]$, a similar inequality may be obtained. However, this inequality reduces to the well known dual observability condition

$$
\operatorname{rank}\left(A^{T} \quad B^{T} A^{T} \quad B^{T}\left(A^{T}\right)^{2} \quad \cdots \quad B^{T}\left(A^{T}\right)^{n-1}\right)^{T}=n,
$$

because all topologies in finite dimensional vector spaces are equivalent. Of course, the above rank condition is exactly the same with the Kalman's rank controllability condition. The above condition is called the observability condition. This duality property is widely known in the finite dimensional linear case (see, for example, Olsder [11] and Wonham [12]). Therefore, we can say that the above inequality is the infinite dimensional version of the Kalman's rank observability condition.

Proposition 2. If $\alpha$ satisfies the following conditions:

A1: There is an $\epsilon \in(0,1)$, such that $|\alpha(t)| \leq(1-\epsilon) \pi^{2}$ for every $t$;

A2: $\operatorname{Var}(\alpha)<\infty$, where $\operatorname{Var}(\alpha)$ denotes the variation of the function $\alpha$ on $(0, \infty)$,

then there exist $T>0$ and $k_{T}, K_{T}>0$ such that

$$
k_{T}\left\|\left(v_{0}, v_{1}\right)\right\|_{H_{0}^{1} \times L^{2}}^{2} \leq\left.\int_{0}^{T}\left(\partial_{x} v(x, t)\right)\right|_{x=1} ^{2} d t \leq K_{T}\left\|\left(v_{0}, v_{1}\right)\right\|_{H_{0}^{1} \times L^{2}}^{2}
$$

for every $\left(v_{0}, v_{1}\right) \in H_{0}^{1}(\Omega) \times L^{2}(\Omega)$.

The proposition above is proved in Pranoto [13] using multiplier technique. This technique is initiated by Komornik [14]. Another important technique that can be used to justify the inequality is micro-local analysis. This technique is utilized by Bardos et al in [15]. Their main contribution is to give sharp conditions on the exact controllability of the conservative wave equations.

Using the inequality (11) and the Hilbert Uniqueness Method (HUM) initiated by Lions [16], one can prove the exact controllability of the system. This is stated in Proposition 3 below. One could also consult Pranoto [17] for proving the exact controllability of time-variant systems utilizing the inequality above. This method reduces the problem of the existence of an exact control to a question whether the inequality (12) is true or not.

Proposition 3. If the assumptions $\mathrm{A} 1$ and $\mathrm{A} 2$ in the above proposition are 
satisfied, then the following system $\boldsymbol{M}$

$$
\begin{aligned}
& L(w)=0 \text { in } \Omega \times(0, T), \\
& w(0, t)=0 \text { and } w(1, t)=\sigma(t), \\
& w(x, 0)=w_{0}(x) \text { and } w_{t}(x, 0)=w_{1}(x)
\end{aligned}
$$

is exact controllable, i.e. there exists a $T>0$ such that for any initial data $\left(w_{0}, w_{1}\right) \in H^{-1} \times L^{2}$ there is a boundary control $\sigma \in L^{2}(0, T)$ such that $w(x, T)=w_{t}(x, T)=0$.

The above proposition means that if the conditions (A1-A2) are satisfied, then for any initial state one can find a control steering it to the equilibrium state in time $T$. So, now we are ready to prove Proposition 1.

Proof of Proposition 1. If we plug $e$ into the system $\mathcal{M}$ above, then it will drive the initial data $(0,0)$ to $\left(w\left(x, T^{\prime}\right)=w_{t}\left(x, T^{\prime}\right)\right)$. By Proposition 3, there exists a $T^{*}>0$, such that for any initial data, we could find a control $f$ driving the initial data to $(0,0)$. In particular, we could find a control $f_{e}$ driving the initial data $\left(w\left(x, T^{\prime}\right)=w_{t}\left(x, T^{\prime}\right)\right)$ to $(0,0)$. Thus, it is clear that the control

$$
\sigma(x, t)=\left(e * f_{e}\right)(x, t)=\left\{\begin{array}{l}
e(x, t) \text { if } 0 \leq t \leq T^{\prime} \\
f_{e}(x, t) \text { if } T^{\prime} \leq t \leq T^{\prime}+T^{*}
\end{array}\right.
$$

steers the zero initial data to itself. So, the function $\sigma$ is a boundary sentinel. If $\boldsymbol{e}$ is not zero, then in general $\boldsymbol{f}_{\boldsymbol{e}}$ is not zero as well. Thus, the concatenate $\boldsymbol{\sigma}=\boldsymbol{e}^{*} \boldsymbol{f}_{\boldsymbol{e}}$ in general is not trivial.

Proof Outline of Proposition 3. First, let $v$ be the solution of the dual system

$$
\begin{aligned}
& L(v)=0 \text { in } \Omega \times(0, T) \\
& v(0, t)=v(1, t)=0 \text { for all } t \\
& v(x, 0)=v_{0}(x) \quad v_{t}(x, 0)=v_{1}(x) .
\end{aligned}
$$


Next, let $p$ be the output function of this dual system, defined by $p(t)=v_{x}(1, t)$. Therefore, for any initial state $\left(v_{0}, v_{1}\right) \in V$, one can obtain a function $p=p(t)$. Next, using this $p$ as the input of the system, one solves the following backward problem

$$
\begin{aligned}
& L(w)=0 \text { in } \Omega \times(0, T) \\
& w(0, t)=0 \text { and } w(1, t)=p(t) \\
& w(\cdot, T)=w_{t}(\cdot, T)=0
\end{aligned}
$$

for calculating the initial conditions $w(\cdot, 0)$ and $w_{t}(\cdot, 0)$. This means that one can construct a linear functional

$$
\begin{aligned}
& \Phi: V \rightarrow V^{\prime} \\
& \Phi:\left(v_{0}, v_{1}\right) \mapsto\left(w(\cdot, 0), w_{t}(\cdot, 0)\right)
\end{aligned}
$$

Now, the exact controllability of course is proved if it can be shown that the above functional is surjective. This is precisely the case, because of the inequality (12). In particular, the left hand inequality of (12) is the main reason on the surjectivity of $\Phi$. The right hand inequality proves the square integrability of the output.

\section{Recovering the Internal Control $u$}

The proof of Proposition 1 implicitly states that one can construct infinitely many boundary sentinels. So, now one may ask how to recover the internal control $u$ using the boundary sentinels.

Now, assume one has a boundary sentinel $\sigma$. Thus, one can construct a linear functional

$$
S\left(\lambda, \tau_{0}, \tau_{1}\right)=\int_{0}^{T} \sigma(t) z\left(t ; \lambda, \tau_{0}, \tau_{1}\right) d t
$$

Since $\sigma$ is a sentinel, the functional $S$ is independent of $\tau_{0}, \tau_{1}$. This implies that for sufficiently small $\lambda$, one may approximate the value of $S\left(\lambda, \tau_{0}, \tau_{1}\right)$ by 


$$
S\left(\lambda, \tau_{0}, \tau_{1}\right) \approx S(0,0,0)+\lambda \partial_{\lambda} S(0,0,0)
$$

Now, if we multiply (3) by $\partial_{\lambda} y$ and integrate it over $\Omega \times(0, T)$, we obtain the following fact

$$
\begin{aligned}
\int_{\Omega \times(0, T)}\left(L(w) \partial_{\lambda} y-L\left(\partial_{\lambda} y\right) w\right) d x d t & =\int_{0}^{T} w(1, t) \partial_{\lambda} y_{x}(1, t) d t \\
& =\partial_{\lambda} \int_{0}^{T} \sigma(t) z(t) d t \\
& =\partial_{\lambda} S(0,0,0) .
\end{aligned}
$$

On the other hand, since $\partial_{\lambda} y$ satisfies the following problem

$$
\begin{aligned}
& L\left(\partial_{\lambda} y\right)=\bar{c} \text { in } \Omega \times(0, T), \\
& \partial_{\lambda} y=0 \text { on } x=0 \text { and } x=1 ; \\
& \partial_{\lambda} y(x, 0)=\partial_{\lambda} y_{t}(x, 0)=0,
\end{aligned}
$$

we obtain

$$
\int_{\Omega \times(0, T)}\left(L(w) \partial_{\lambda} y-L\left(\partial_{\lambda} y\right) w\right) d x d t=-\int_{\omega \times(0, T)} w \bar{c} d x d t .
$$

Thus, the relations (15), (16), (17) provide the main relation between $S$ and $\bar{c}$ :

$$
S\left(\lambda, \tau_{0}, \tau_{1}\right)-S(0,0,0) \approx-\lambda \int_{\omega \times(0, T)} w \bar{c} d x d t
$$

The values $S\left(\lambda, \tau_{0}, \tau_{1}\right)$ and $S(0,0,0)$ are known from observations. This implies that the left hand side of the above relation is known. Therefore, the relation above means that one can learn about the internal control $u=c+\lambda \bar{c}$ from the value $\lambda \int_{\omega \times(0, T)} w \bar{c} d x d t$. More specifically, the effect of the perturbation $c$ can be studied from the values $S\left(\lambda, \tau_{0}, \tau_{1}\right)-S(0,0,0)$. Of course, the relation (18) cannot be used to completely recover the exact $\bar{c}$ which is the perturbation of $u$, if one uses only one $w$. However, the relation (18) can provide some information about $\bar{c}$ if one can generate a sufficient number of $w$ 's that may form a kind of basis of the space $L^{2}(\omega \times(0, T))$. Thus, analogous to Fourier transformation that converts the information of the 
signal to the set of Fourier coefficients, here (18) provides some partial information about $\bar{c}$.

\section{$4 \quad$ Future Works}

Some future work that we are interested to pursue is to design a systematic method for constructing an almost complete set of boundary sentinels. This method will be very useful for making the sentinel method above efficient and effective for recovering the internal control.

\section{Acknowledgments}

The author is very grateful for the comments provided by the anonymous referees. Their recommendations are valuable.

This work is partially supported by the $14^{\text {th }}$ Competitive Grant (Hibah Bersaing XIV), provided by the Minister of National Education, Republic of Indonesia. The author thanks the Minister for the support.

\section{References}

[1] Lions, J.L., Sentinels and Stealthy Perturbations. Semi Complete Set of Sentinels, Math. and Numerical Aspects of Wave Propagation Phenomena, by G. Cohen (eds), SIAM Philadelphia, pp. 239-251, 1991.

[2] Lions, J.L., Sentinelles pour Les Systemes Distribues, Masson, Paris, 1992.

[3] Pranoto, Internal Control Recovery on Klein-Gordon Systems, J. Indones. Math. Soc., 10(2), 115-124, 2004.

[4] Pranoto, A Brief Summary on the Control Recovery of Time-Varying K-G Systems, International Journal of Modelling, Identification and Control, 8(1), 2009.

[5] Zuazua, E., Exact Controllability of Semilinear Wave Equations in One Space Dimension, Ann. Inst. H. Poincare Anal. Non Lineaire, 10, 109 129, 1993.

[6] Zhang, X. \& Zuazua, E., Exact Controllability of The Semi-linear Wave Equations, in Sixty Open Problems in the Mathematics of Systems and Control, ed by V.D. Blondel and A. Megretski, Princeton Univ. Press, 2003.

[7] Zuazua, E., Propagation, Observation, and Control of Waves Approximated by Finite Difference Methods, SIAM Review, 47 (2), 197243, 2005.

[8] Zuazua, E., Control and Numerical Approximation of the Wave and Heat Equations, Proceedings of the ICM 2006, Vol. III, "Invited Lectures", 
European Mathematical Society Publishing House, M. Sanz-Solé et al. eds., pp. 1389-1417, 2006.

[9] Acheli, D., Kernevez, J.P. \& Oukaci, F., The Sentinel Method Used in Identification of The Position and Trajectory of A Source of Pollution, Appl. Anal. 70(3-4), 303-319, 1999.

[10] Fliess, M., Join, C. \& Sira-Ramirez, H., Non Linear estimation Is Easy, International Journal of Modelling, Identification and Control, 4(1), 12 27, 2008.

[11] Olsder, G.J., Mathematical Systems Theory, Delft University Press, 1994.

[12] Wonham, W.M., Linear Multivariable Control: A Geometric Approach, Applications of Mathematics, 10, Springer-Verlag, 1979.

[13] Pranoto, An Equality of the 1-D Klein-Gordon Equation with A TimeVarying Parameter, Journal of Inequality in Pure and Applied Mathematics, Art. 45, 3(3), 2002.

[14] Komornik, V., Exact Controllability and Stabilization: The Multiplier Method, Research in Applied Mathematics, Masson, 1994.

[15] Bardos, C., Lebeau, G. \& Rauch, J., Sharp Sufficient Conditions for the Observation, Control, and Stabilization of Waves from the Boundary, SIAM J. Control and Optimization, 30(5), 1024-1065, 1992.

[16] Lions, J.L., Controllabilite Exacte Perturbations et Stabilisation de Systemes Distribues, Masson, Paris, 1988.

[17] Pranoto, I., Exact Controllability of Klein-Gordon Systems with A Timevarying Parameter, in Topics in Applied and Theoretical Mathematics and Computer Science, edited by V.V. Kluev, et al, World Scientific and Engineering Academy and Society, 76 - 80, 2001. 